\title{
Proper Hand Washing for Caregivers
}

Hand washing is an important way to reduce the fecal-oral transfer of germs that cause diseases. Young children and frail elders have less ability to fight germs than others. As a caregiver you can help reduce the risk of diseases that can be transmitted in a crowded environment by using proper hand washing procedures.
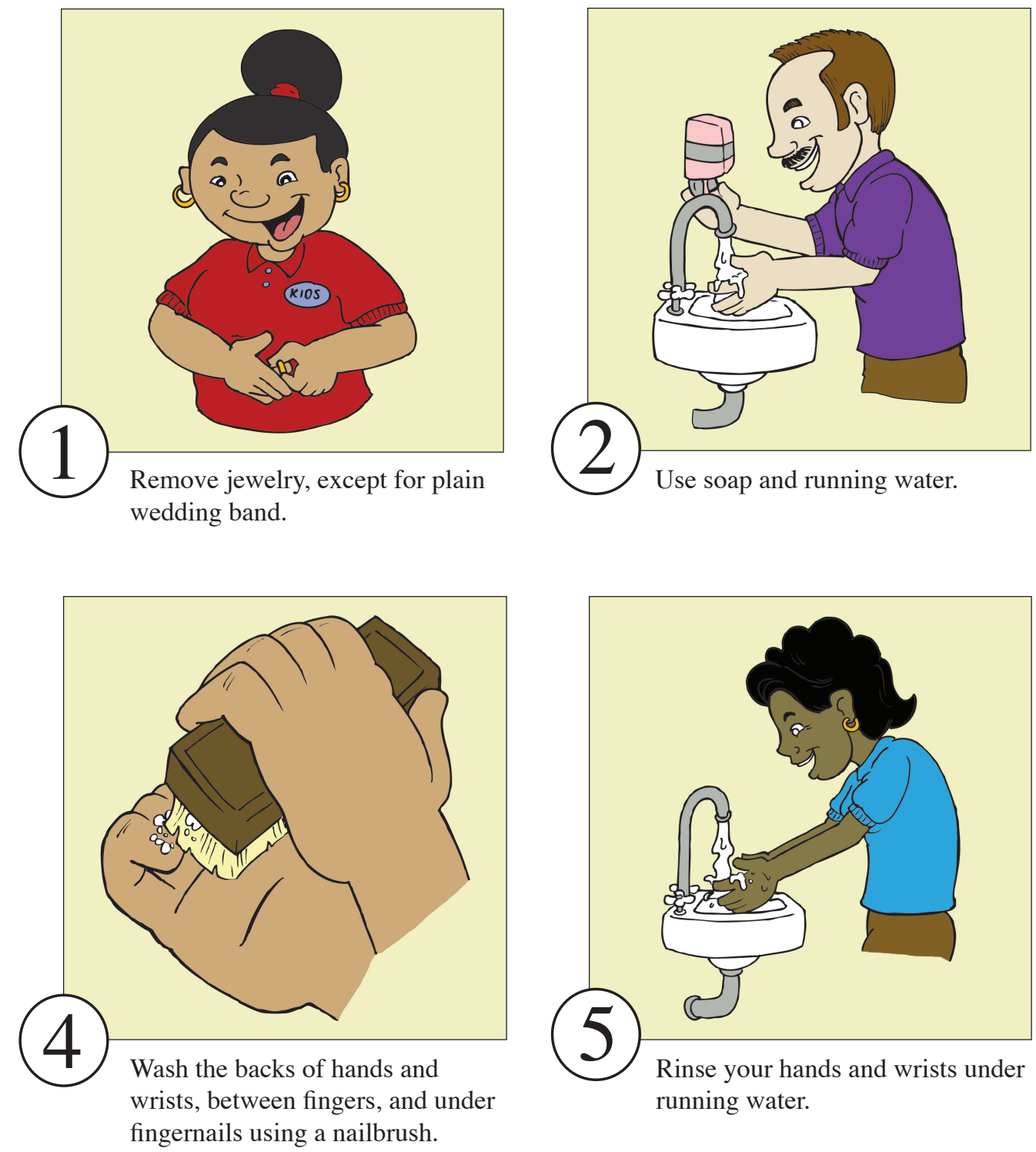

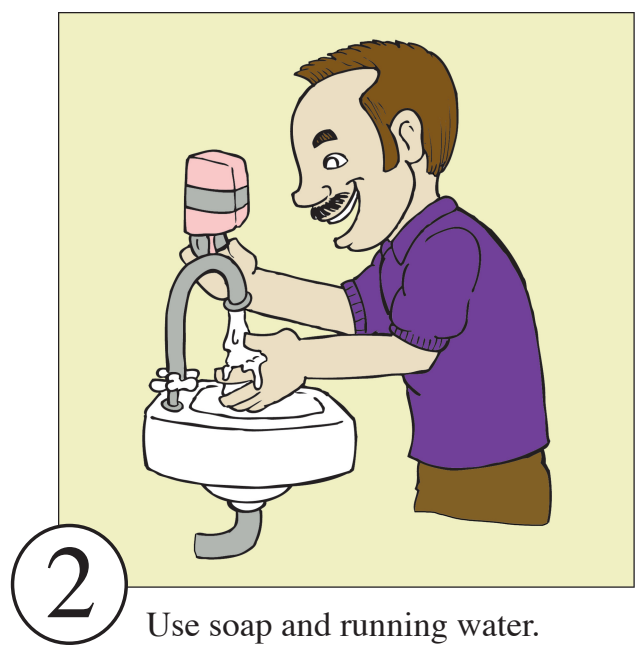

running water.
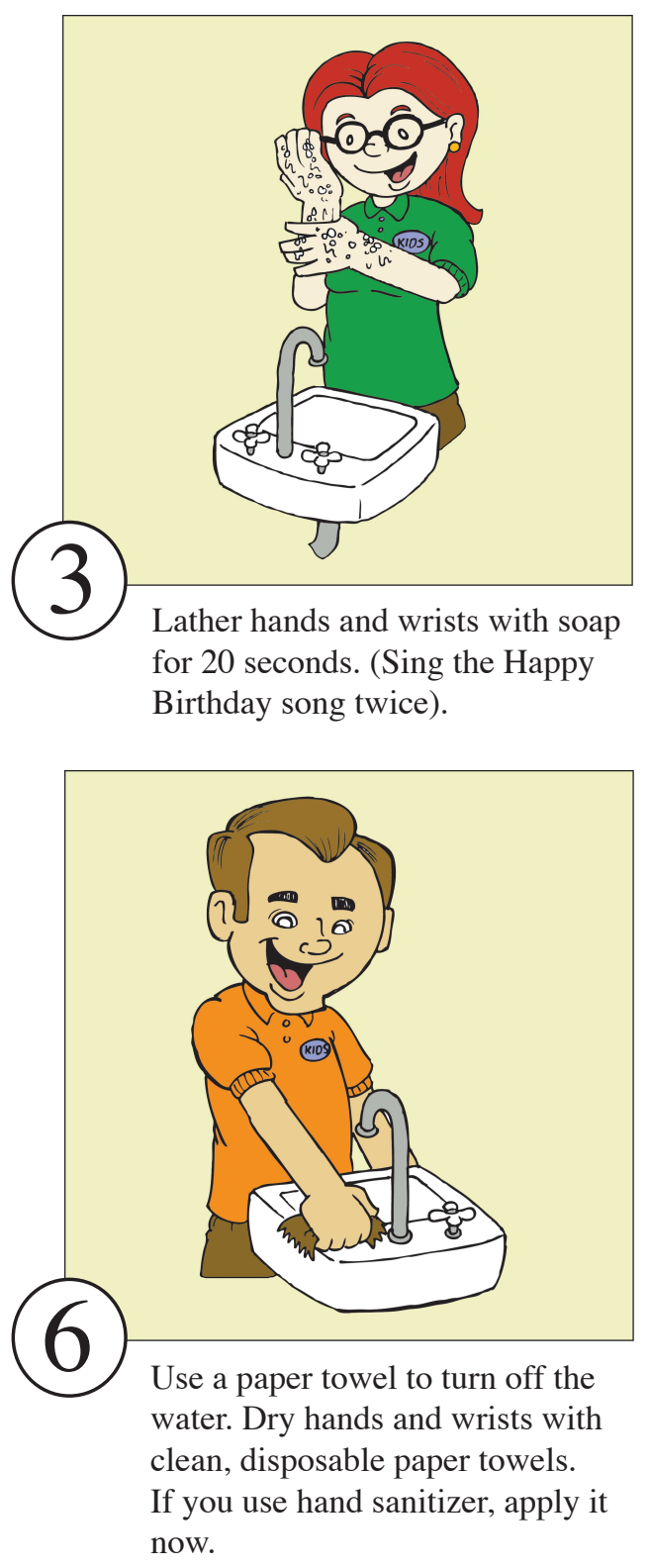

Lather hands and wrists with soap for 20 seconds. (Sing the Happy Birthday song twice). now.

\section{Wash your hands before:}

- Eating and preparing foods

- Touching serving utensils

- Preparing formula for infants

- Handling ready to eat foods

- Serving foods 